\title{
Alum Augments the Experimental Allergenicity of Kunitz-Type Soybean Trypsin Inhibitor Independent of the Antigen-Adsorption
}

\author{
Rintaro YAMANISHI, Ikuko YusA, Akiko MIYAMOTO, Izumi SATO, Noriko BANDO and \\ Junji TERAO \\ Department of Nutrition, School of Medicine, The University of Tokushima, Kuramoto-cho 3-18-15, \\ Tokushima, Tokushima 770-8503, Japan
}

(Received May 20, 2003)

\begin{abstract}
Summary In order to inspect the significance of the adsorbing property in the adjuvant activity to enhance IgE production, we immunized BALB/c mice against Kunitz-type soybean trypsin inhibitor (KSTI), the most potent experimental allergen among soybean proteins, associated with Aluminum hydroxide (alum) or DEAE-Sephadex particles. The production of immunoglobulin isotypes was analyzed at the various amounts, 3-3,000 $\mu \mathrm{g}$ per mouse, of the antigen dosages. In our experiments, although alum did not adsorb KSTI significantly, it augmented the total and the antigen-specific IgE without affecting the optimal range of the antigen dosage. On the other hand, alum did not effectively enhance the production of the other immunoglobulin isotypes. The production of immunoglobulin isotypes other than IgE increased dose-dependently on the antigen. These results ensured our previous finding that another protein, ovalbumin, was used as the antigen. We also demonstrated that the adsorption of KSTI by DEAE-Sephadex in the immunizing vehicle resulted in the requirement of more KSTI for accomplishing the equal immunity in BALB/c mice compared to the control. Moreover, we demonstrated that, regardless of the inability to adsorb KSTI, alum exerted its adjuvant activity only when it was co-injected with the antigen. These results showed that some biochemical effect, other than adsorptive activity, to enhance the production of the antigen-specific IgE resides in alum.
\end{abstract}

Key Words alum, Kunitz-type soybean trypsin inhibitor, adjuvant, IgE

The crosslinking among high affinity IgE receptors on mast cell or basophil, via IgE bound to the responsible allergen, is a trigger for type-I allergic disease (1). Thus to investigate how IgE is produced or how the production is augmented is essential for putting on the brakes to the prevalence of allergic disease among developed countries.

An adjuvant is the substance that augments immunity and may affect the balance between type I helper $\mathrm{T}$ lymphocytes (Th1-type) immunity and type II helper T lymphocytes (Th2-type) immunity. For example, oligodeoxynucleotides containing the $\mathrm{CpG}$ motif, which is naturally present in bacterial DNA, have been suggested as an adjuvant to induce Th1-type immunity (2), whereas diesel exhaust particulate (DEP), an air pollutant, has been suggested as an adjuvant to enhance Th2-type (3). Aluminum hydroxide (alum) has often been used under experimental conditions to produce antigen specific $\operatorname{IgE}$ in murine cases (4), which suggested that alum was an adjuvant stimulating Th2-type immunity. Although the adjuvant activity of alum is partly explained by its antigen-adsorbing property (5) to make antigen presentation efficient, its function, especially how to evoke the IgE production, is not yet fully understood.

Previously we demonstrated that, when ovalbumin

E-mail: rintaro@nutr.med.tokushima-u.ac.jp was used as an antigen to $\mathrm{BALB} / \mathrm{c}$ mice, the adjuvant activity of alum did not accompany with the alternation of the optimal dosage of the injected antigen (6). This suggests that the adjuvant activity of alum do not participate in the antigen presentation. In this report, we performed immunization against the other experimental antigen, Kunitz-type soybean trypsin inhibitor (KSTI), not only to ensure the previous result but also to clarify the relation between the adsorbing property and the adjuvant activity.

\section{MATERIALS AND METHODS}

Animals. Female BALB/c mice, 8 wk old, and female Wistar rats, 15 wk old, were purchased from SLC Japan (Hamamatsu, Japan). The animals were housed in our animal room at about $25^{\circ} \mathrm{C}$ with adequate humidity, and fed with a standard pellet diet and water ad lib. All experiments were performed in accordance with the Guidelines for Animal Experimentation (Japanese Association for Laboratory Animal Science, 1987).

Particles. Aluminum hydroxide (Nacalai Tesque, Inc., Kyoto, Japan) was ground in a mortar thoroughly for using it as an alum adjuvant. DEAE-Sephadex (Amersham Pharmacia Biotech, Buckinghamshire, UK) was incubated overnight with sterile phosphate-buffered saline (PBS) at $4^{\circ} \mathrm{C}$. PBS was removed just before use.

Adsorptive property. To determine the adsorptive 
property of alum or DEAE-Sephadex to KSTI, those particles were incubated overnight with KSTI at $4^{\circ} \mathrm{C}$. After the centrifugation $\left(300 \times \mathrm{g}\right.$ for $10 \mathrm{~min}$, at $\left.4^{\circ} \mathrm{C}\right)$, the supernatants were filtrated with $0.8 \mu \mathrm{m}$ membrane filter (Millipore, Billerica, MA, USA). The adsorptive property for KSTI was determined by measuring protein concentration of filtrates. Protein concentration was evaluated by the method of Lowry et al. (7).

Immunization. Three times with $2 \mathrm{wk}$ interval $3 \mu \mathrm{g}$, $30 \mu \mathrm{g}, 300 \mu \mathrm{g}$ or $3 \mathrm{mg}$ KSTI (Sigma; St Louis, MO, USA) per mouse, was subcutaneously, intraperitoneally in a special case, injected into $\mathrm{BALB} / \mathrm{c}$ mice with or without alum (2 mg) in $400 \mu \mathrm{L}$ of PBS. Each group contained 5 or 6 mice. Whole anti-sera were collected $1 \mathrm{wk}$ after the third immunization and stored at $-20^{\circ} \mathrm{C}$ until use.

In one experiment we used DEAE-Sephadex particles ( $2 \mathrm{mg}$ ) in place of alum. In this experiment, the DEAESephadex particles were pre-incubated with KSTI. After the removal of free KSTI, they were injected with normal mice sera to BALB/c mice. For the control experiment, DEAE-Sephadex particles pre-incubated with normal mice sera were injected to BALB/c mice just after mixed with KSTI.

Passive cutaneous anaphylaxy (PCA) reaction. PCA reactions were carried out as previously described (8) In brief, Wistar rats anesthetized by Nembutal (Abbott, Chicago, IL, USA) were intracutaneously injected with diluted anti-sera into skin on the back. After $2 \mathrm{~d}$, the rats were again anesthetized and intravenously injected with $1 \mathrm{mg}$ of OVA and $0.5 \%$ of Evans Blue (Nacalai Tesque) in $1 \mathrm{~mL}$ of PBS. Photographs of the resulting back skins of rats that expressed blue wheal spots were taken by an MP4 Instant Camera System (Polaroid, Cambridge, MA, USA).

Enzyme-linked immunosorbent assay (ELISA). Total amounts of IgE were measured by Sandwich ELISA kits (Bethyl, Montgomery, TX. USA). Titers of the OVA-specific immunoglobulin isotype were evaluated by ELISA with the use of antigen-coated micro-plates and detecting antibodies for IgG1, IgG2a or IgM (Bethyl), respectively. The protocol of the ELISA has been already described (9). In brief, plates coated with KSTI or the capture-antibody were incubated with BSA solution for $1 \mathrm{~h}$ at room temperature (RT) for blocking, with diluted anti-sera for $1 \mathrm{~h}$ at RT, washed 3 times with PBS containing $0.05 \%$ Tween 20 , and then incubated with adequate horse radish peroxidase-labeled antibody for $1 \mathrm{~h}$ at RT for detection. After again washing 3 times with PBS containing $0.05 \%$ Tween 20, the plates were devel- (a)

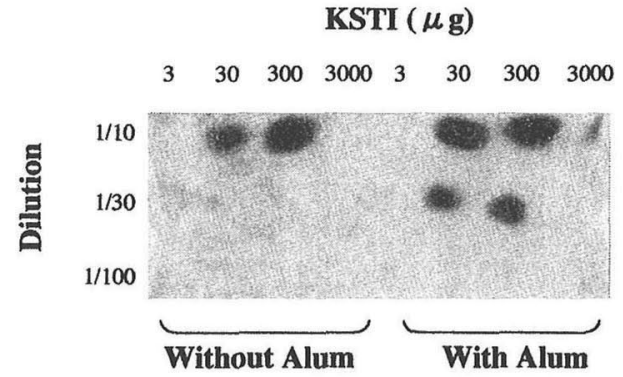

(c)

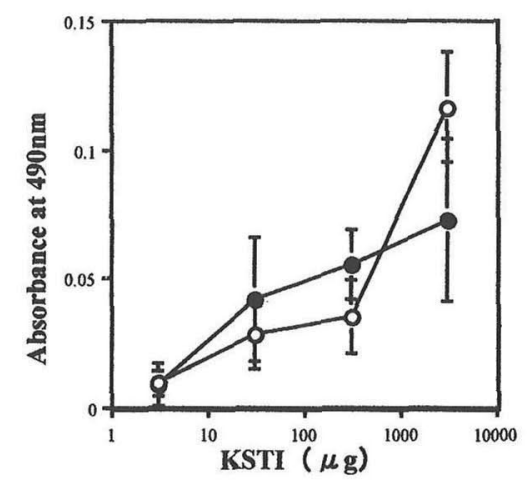

(b)

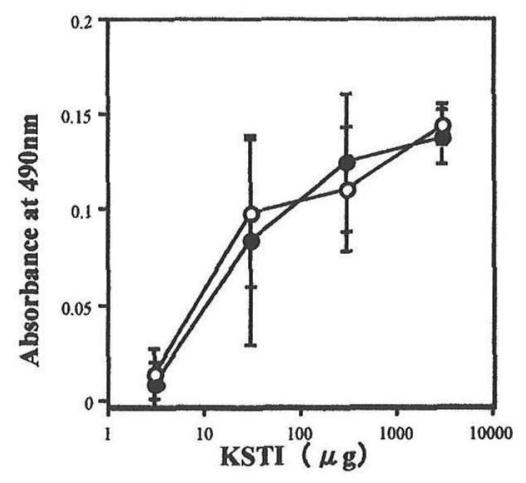

(d)

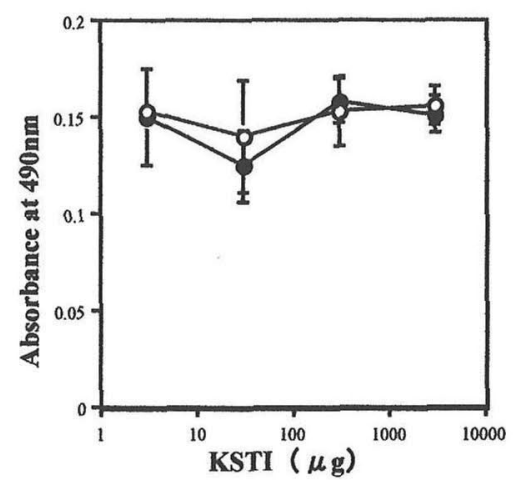

Fig. 1. The production of KSTI-specific Immunoglobulins in BALB/c mice immunized by various doses of KSTI with or without alum. Sera from BALB/c mice immunized by designated doses of KSTI with or without alum were analyzed. To evaluate the antigen specific IgE titer of mice sera, PCA reaction was proceeded in a Wistar rat which was intracutaneously injected with anti-sera diluted by PBS into the back skin, followed by the intravenous injection of the antigen and a pigment after $2 \mathrm{~d}$ (a). To evaluate the antigen specific IgG1 (b), IgG2a (c), and IgM (d), in the sera immunized by KSTI with $(\bullet)$ or without $(O)$ alum, the antigen-coated 96 well micro-plate was incubated with the immunized sera, which were diluted $1: 200$ for IgG1, 1:50 for IgG2a, and 1: 50 for IgM, followed by the incubation with the enzyme-labeled responsible second antibody. The immunocomplex on the wells were incubated with 0 -phenylenediamine $(0.4 \mathrm{mg} / \mathrm{mL})$ and $0.01 \%$ $\mathrm{H}_{2} \mathrm{O}_{2}$ as the substrate. The absorbance of the reaction mixture in the wells at $492 \mathrm{~nm}$ were determined. Values are expressed as mean \pm SD. 
(a)

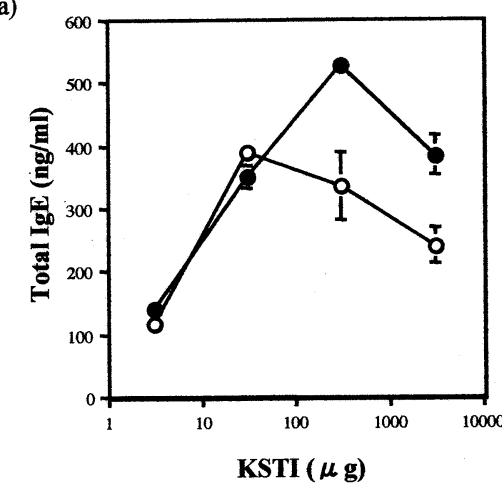

(b)

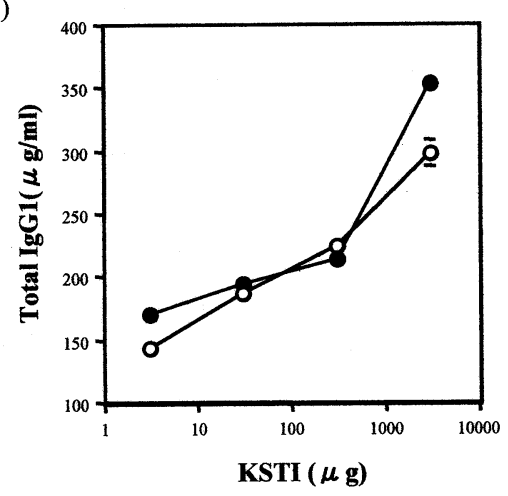

Fig. 2. The production of Th2-type immunoglobulin isotypes in BALB/c mice immunized with various doses of the antigen. Sera from BALB/c mice immunized by designated doses of KSTI with $(\bullet)$ or without $(\bigcirc)$ alum were analyzed. To evaluate the concentration of serum total IgE (a) or IgG1 (b), the sandwich ELISA was proceeded with a 96 well microplate coated with anti mouse IgE or IgG1. The plates were incubated with the immunized sera, followed by the incubation with the enzyme-labeled responsible second antibody. The immunocomplex on the wells were incubated with o-phenylenediamine $(0.4 \mathrm{mg} / \mathrm{mL})$ and $0.01 \% \mathrm{H}_{2} \mathrm{O}_{2}$ as the substrate. The absorbance of the reaction mixture in the wells at $492 \mathrm{~nm}$ were determined. The concentration was determined based on a standard curve of IgE or IgG1. Values are expressed as mean \pm SD.

oped with o-phenylenediamine and hydrogen peroxide. The absorbance of each well was measured by a Model 450 micro-plate reader (Bio-Rad, Hercules, CA, USA).

\section{RESULTS}

The production of antigen specific immunoglobulin in $\mathrm{BALB} / \mathrm{c}$ mice immunized with various dosages of KSTI with or without alum was analyzed by PCA reaction and ELISA. As for the IgE production the suitable dosages of the injected antigen, which ranged from 30 to $300 \mu \mathrm{g}$ dosage per mouse, were not influenced by the presence of alum (Fig. 1a). This meant that alum did not up-regulate the efficiency of the antigen presentation. On the other hand, alum actually augmented the titer of the antigen-specific IgE at those suitable doses.

Titers of KSTI specific IgG1, IgG2a and IgM were analyzed by the antigen-specific ELISA, with plates coated by KSTI (Fig. 1b, c, d). Different from IgE, the titer of antigen-specific IgG1 and IgG2a increased in the antigen-dose dependent manner. The production of the antigen specific IgM did not respond to the antigen dose. Alum did not enhance the immunoglobulin isotypes other than IgE significantly.

As the production of IgE and IgG1 are both induced by Th2, their serum total concentrations were also measured by a sandwich ELISA (Fig. 2). The results showed almost consistent pattern with the antigen-specific activities. Namely, not the concentration of serum total IgG1 but that of serum total IgE was augmented by the presence of alum at immunization. As for the relation with antigen-dosage, that of IgG1 increased in the dose dependent manner, whereas that of $\operatorname{IgE}$ had a peak within the range we tested.

It has been believed that the adjuvant activity of alum is exerted via its adsorbing activity to enhance the efficiency of the antigen presentation (5). To access the actual property that brings the adjuvant activity, we investigated the adsorptive activity of alum in our experimental condition and compared it with that of
Table 1. The adsorption of KSTI by alum or DEAESephadex.

\begin{tabular}{ccc}
\hline \multirow{2}{*}{$\begin{array}{c}\text { Amount of KSTI } \\
(\mu \mathrm{g})\end{array}$} & \multicolumn{2}{c}{ Adsorption efficiencies (mean \pm SD $\%)$} \\
\cline { 2 - 3 } & Alum & DEAE-Sephadex \\
\hline 30 & $4.0 \pm 4.11$ & $66.0 \pm 2.94$ \\
300 & $3.5 \pm 3.44$ & $68.0 \pm 2.16$ \\
3,000 & $1.1 \pm 0.35$ & $71.0 \pm 0.82$ \\
\hline
\end{tabular}

Alum or DEAE-Sephadex particles $(2 \mathrm{mg})$ were incubated with a designated amount of KSTI at $4^{\circ} \mathrm{C}$ overnight in a rotating cultivator. The remaining amount of the protein in the supernatant was measured and the percentage of the adsorbed protein was determined by subtraction from the total amount. The values expressed as adsorbing efficiencies are calculated from experiments repeated five times for alum and in triplicate for DEAESephadex.

DEAE-Sephadex particles (Table 1). The adsorption of KSTI to the particles was determined by measuring the residual amount of the protein in the supernatants after the incubation with alum or DEAE-Sephadex. More than $95 \%$ of KSTI was recovered from the supernatant of alum and, on the other hand, less than $40 \%$ was recovered from that of DEAE-Sephadex. We regarded the small percentage of alum-adsorbed KSTI as the loss of recovery. Thus the table showed that, different from DEAE-Sephadex, alum did not adsorb KSTI practically. In order to inspect whether the adsorption is involved in the adjuvant activity that brings the IgE-hyperproduction, we used DEAE-Sephadex particles instead of alum at immunizing BALB/c mice by KSTI. The adsorption by DEAE-Sephadex resulted in less sensitive production of antigen-specific IgE to the smaller dose, 3-30 $\mu \mathrm{g}$, of the antigen (Fig. 3).

We finally investigated a sphere of the adjuvant activity of alum. For this purpose we separately injected 
KSTI $(\mu \mathrm{g})$

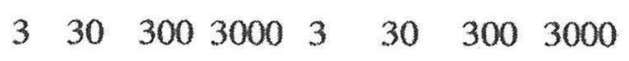

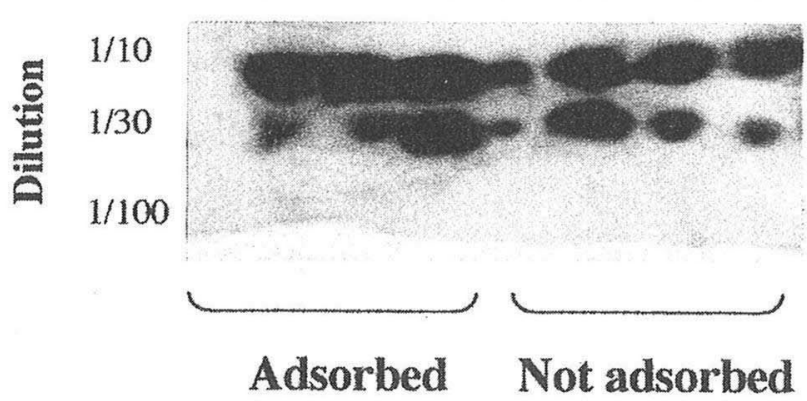

Fig. 3. The production of KSTI-specific IgE in BALB/c mice immunized by various doses of the antigen adsorbed or not adsorbed by DEAE-Sephadex. Sera from $\mathrm{BALB} / \mathrm{c}$ mice immunized by designated doses of KSTI adsorbed or not adsorbed by DEAE-Sephadex were analyzed. DEAE-Sephadex particles incubated with KSTI, followed by the addition with normal sera of BALB/c mice, were prepared for the immunization with adsorbed KSTI and DEAE-Sephadex particles incubated with normal sera, prior to the addition with KSTI, were prepared for the immunization with not adsorbed KSTI. Two mg of DEAE-Sephadex was injected into each mouse. In adsorbed KSTI immunization, overloaded (not adsorbed) KSTI present in the supernatant were removed and the injected amounts of KSTI were adjusted as designated with the consideration of the adsorbing efficiency shown in Table 1. Titers of IgE were evaluated by PCA reaction described in Fig. 1.

\section{KSTI Alum}

\section{Dilution}

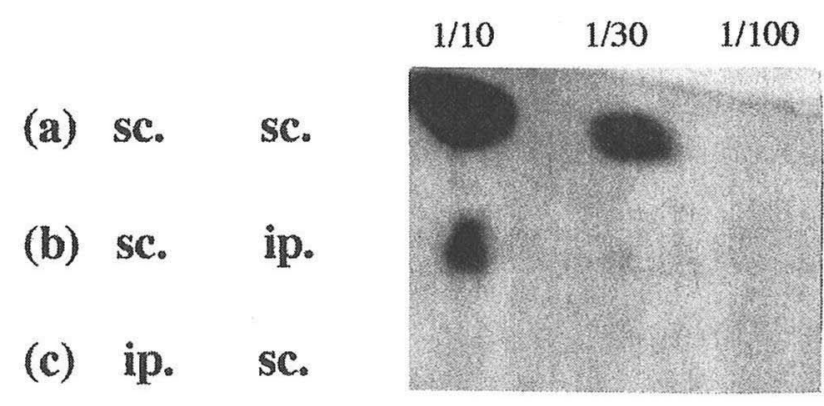

Fig. 4. The production of KSTI-specific IgE in BALB/c mice responded to the injected portion of the antigen and alum. Sera from BALB/c mice immunized by KSTI with co-injected alum or separately injected alum were analyzed. KSTI was injected under the back skin of BALB/c mice with alum (a). KSTI was injected under the back skin but alum was injected ip. (b), or KSTI was injected ip. but alum was injected under the back skin (c). Titers of IgE were evaluated by PCA reaction described in Fig. 1.

alum from the antigen. When alum was injected intraperitoneally (ip.) and the antigen was subcutaneously (sc.) injected the back of BALB/c mice, the production of $\operatorname{IgE}$ specific for the antigen was remarkably attenuated compared with the case of sc. co-injection to the back (Fig. 4). Moreover when alum was sc. injected the back and the antigen was injected ip., the production of IgE (a)

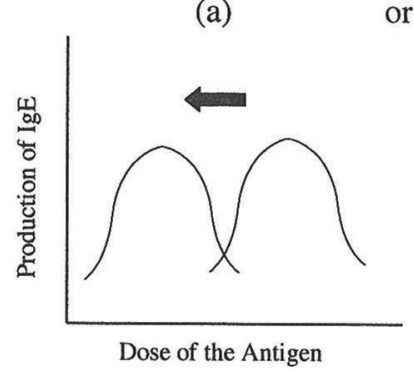

or

(b)

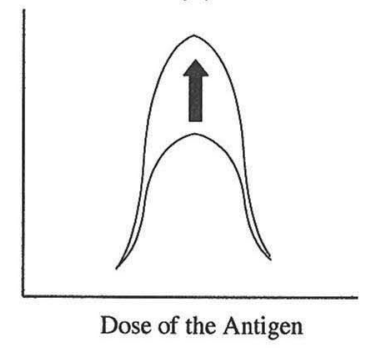

Fig. 5. The alternative kinetics of IgE production stimulated by an adjuvant. The effect of the adjuvant is shown as an arrow. (a) The case if the sensitivity for IgE production to the dose of the antigen would be enhanced by an adjuvant. (b) The case if the degree of the IgE production would be enhanced by an adjuvant.

specific for the antigen was under the detection limit.

\section{DISCUSSION}

Consistent with our previous results obtained by the immunization against a different protein, ovalbumin (6), alum did not influence the sensitivity of the IgE production to the antigen KSTI dosage, but enhanced the antigen-specific titer and the serum total concentration of IgE at the optimal dosages. This suggests that the adjuvant activity of alum is independent of the antigen presentation efficiency. If the activity were dependent on it, the optimal dosage of antigen for inducing the antigen-specific IgE must have shifted to the lower side as shown in Fig. 5A, in accordance with the adjuvant use. As a matter of fact, the adjuvant activity resulted in the pattern of Fig. 5B.

The adjuvant activity of alum has been explained by its adsorptive property that may effect on the antigenpresentation. Conflictingly alum did not practically adsorb KSTI but did work as an effective adjuvant to the IgE in our experiments. Strictly speaking, it is impossible to deny the presence of the subtle adsorption by alum, which might contribute to the production of $\operatorname{IgE}$. Thus, alternatively we immunized BALB/c mice against KSTI using an adsorptive particle, DEAE-Sephadex, for clarifying the relation between the antigen-adsorption and the production of IgE. The adsorption of KSTI by DEAE-Sephadex particle resulted in the requirement of more KSTI for accomplishing equal IgE production compared to the control, suggesting that the antigenadsorption by a particle did not contribute to it. This strengthened our findings about alum that its adjuvant activity to augment IgE production is independent of the antigen adsorption.

Whereas alum did not adsorb the antigen in our injected solution, it requires the co-injection with the antigen to bring its adjuvant activity. This result revealed that the sphere of the adjuvant activity of alum was not systemic but local, in other words, limited to the portion where the antigen was injected. This suggests that only the cells that encounter both the antigen and the alum are affected, the most possible candidate of which may be the antigen-presenting cell. Such limitation might be a reason for the misunderstanding that 
the antigen-adsorption by alum would be necessary for its adjuvant activity.

The property of the adjuvant to enhance IgE production is still under controversy. The effect of a physical property, that is, the size of a lipid vesicle to entrap the antigen on the adjuvant activity was reported: the larger $(\geq 225 \mathrm{~nm})$ vesicle induced a Th1 response and the smaller $(\leq 155 \mathrm{~nm}$ ) vesicles induced a Th2 response (10). On the other hand, a recent report concerning DEP, which has been reported to enhance IgE production, suggests that its biochemical property, that is, prooxidant activity is included in its activities exhibited in the immune system (11). Aluminum is not an oxidative element by itself, but it has been reported that aluminum was involved in redox reactions under the experimental conditions (12). Similar with DEP, redoxrelated activity of aluminum may contribute to the adjuvant activity. Consistent with this hypothesis, our recent results demonstrated that the intake of anti-oxidative vitamins by BALB/c mice resulted in the attenuation of IgE production (13). Concerning the mechanism of alum's adjuvant activity, the IL4- or IL13-independent adjuvant activity to initiate the antigen-specific Th2 response was reported (14), however, its essential property and the cell subjected to the direct influence from it remains to be elucidated.

Aluminum is a common but, in a sense, quite unique element. Though it is the third abundant element on earth, no beneficial biological property has been known about it. On the contrary it is well known that aluminum accumulates in the brain of Alzheimer disease. In spite of such undesirable evidences, alum has been used as an adjuvant not only to experimental animals but also to humans in artificial immunization. If the adjuvant activity of alum is not due to its adsorptive property, injected alum may unexpectedly influence the immunity of other substances in addition to injected antigens. Moreover, environmental aluminum, including aluminum tableware and cans, may affect natural sensitization to allergens as well.

In conclusion, our experiments indicated that alum had the adjuvant activity independent of antigenadsorption. This finding may be important to reveal the intrinsic property to cause hyperproduction of IgE.

\section{REFERENCES}

1) Turner H, Kinet JP. 1999. Signaling through the highaffinity IgE receptor Fc epsilon RI. Nature 402 (6760
Suppl): B24-30.

2) Krieg AM. 2002. CpG motifs in bacterial DNA and their immune effects. Annu Rev Immunol 20: 709-760.

3) Diaz-Sanchez D, Tsien A, Fleming J, Saxon A. 1997. Combined diesel exhaust particle and ragweed allergen challenge markedly enhance human in vivo nasal ragweed-specific IgE and skews cytokine production to a T helper cell 2-type pattern. J Immunol 158: 2406-2413.

4) Gupta RK, Rost, BE, Relyveld E, Siber GR. 1995. Adjuvant properties of aluminum and calcium compounds. Pharm Biotechnol 6: 229-248.

5) Report of a WHO Scientific Group. 1976. Immunological adjuvants. World Health Organ Tech Rep Ser 595: 140.

6) Yamanishi R, Yusa I, Bando N, Terao J. 2003. Adjuvant activity of alum in inducing antigen specific IgE antibodies in BALB/c mice: a reevaluation. Biosci Biotechnol Biochem 67: 166-169.

7) Lowry OJ, Rowebrough NJ, Farr AL, Randall RJ. 1951. Protein measurement with the Folin phenol reagent. $J$ Biol Chem 193: 265-275.

8) Yamanishi R, Kondo K, Tsuji H, Ogawa T. 1995. Microassay to measure the allergenicity of a Kunitz-type soybean trypsin inhibitor toward Balb/c mice by using RBL-2H3 cells. Biosci Biotechnol Biochem 59: 12721275.

9) Tsuji H, Okada N, Yamanishi R, Bando N, Kimoto M, Ogawa T. 1995. Measurement of Gly m Bd 30K, a major soybean allergen, in soybean products by a sandwich enzyme-linked immunosorbent assay. Biosci Biotechnol Biochem 59: 150-151.

10) Brewer JM, Tetley L, Richmond J, Liew FY, Alexander J. 1998. Lipid vesicle size determines the Th1 or Th2 response to entrapped antigen. J Immunol 161: 40004007.

11) Casillas AM, Hiura T, Li N, Nel AE. 1999. Enhancement of allergic inflammation by diesel exhaust particles: permissive role of reactive oxygen species. Ann Allergy Asthma Immunol 83: 624-629.

12) Yoshino M, Ito M, Haneda M, Tsubouchi R, Murakami K. 1999. Prooxidant action of aluminum ion-Stimulation of iron-mediated lipid peroxidation by aluminum. BioMetals 12: 237-240.

13) Bando N, Yamanishi R, Terao J. 2003. Supplementation of vitamin $\mathrm{E}$ with $\beta$-carotene effectively inhibits immunoglobulin E production in allergic model mice. Biosci Biotechnol Biochem 67: 2176-2182.

14) Brewer JM, Conacher M, Hunter CA, Mohrs M, Brombacher F, Alexander J. 1999. Aluminium hydroxide adjuvant initiates strong antigen-specific Th2 response in the absence of IL-4- or IL-13-mediated signaling. J Immunol 163: 6448-6454. 